\title{
Clinical outcomes after IL-6 blockade in patients with COVID-19 and HIV: a case series
}

\author{
Samuel J. Minkove ${ }^{1,2 \dagger}$, Grant Geiger ${ }^{3}$, Josep M. Llibre ${ }^{4}$, Mary W. Montgomery ${ }^{5}$, Natalie E. West ${ }^{1}$, \\ Natasha M. Chida ${ }^{1}$, Annukka A. R. Antar ${ }^{1 \dagger}$, Dima Dandachi ${ }^{6 \dagger}$, Ethel D. Weld ${ }^{1 *+}$ and HIV-COVID-19 Consortium
}

\begin{abstract}
Background: In hospitalized people with HIV (PWH) there is an increased risk of mortality from COVID-19 among hospitalized PWH as compared to HIV-negative individuals. Evidence suggests that tocilizumab-a humanized monoclonal interleukin (IL)-6 receptor inhibitor (IL-6ri) antibody — has a modest mortality benefit when combined with corticosteroids in select hospitalized COVID-19 patients who are severely ill. Data on clinical outcomes after tocilizumab use in PWH with severe COVID-19 are lacking.

Case presentation: We present a multinational case series of $18 \mathrm{PWH}$ with COVID-19 who were treated with IL-6ri's during the period from April to June 2020. Four patients received tocilizumab, six sarilumab, and eight received an undocumented IL-6ri. Of the 18 patients in the series, 4 (22\%) had CD4 counts $<200$ cells $/ \mathrm{mm}^{3} ; 14$ (82\%) had a suppressed HIV viral load. Eight patients (44\%), all admitted to ICU, were treated for secondary infection; 5 had a confirmed organism. Of the four patients with CD4 counts $<200$ cells $/ \mathrm{mm}^{3}$, three were treated for secondary infection, with 2 confirmed organisms. Overall outcomes were poor-12 patients (67\%) were admitted to the ICU, 11 (61\%) required mechanical ventilation, and 7 (39\%) died.

Conclusions: In this case series of hospitalized PWH with COVID-19 and given IL-6ri prior to the common use of corticosteroids, there are reports of secondary or co-infection in severely ill patients. Comprehensive studies in PWH, particularly with CD4 counts $<200$ cells, are warranted to assess infectious and other outcomes after IL-6ri use, particularly in the context of co-administered corticosteroids.
\end{abstract}

\section{Background}

The interplay of COVID-19 and HIV is not completely understood [1]. However, a recent report by the World Health Organization (WHO) found that HIV was independently associated with a higher risk of in-hospital COVID-19 mortality, even after adjusting for age, sex,

\footnotetext{
*Correspondence: eweld@jhmi.edu

†'Samuel J. Minkove, Annukka A. R. Antar, Dima Dandachi and Ethel D. Weld contributed equally

1 Department of Medicine, The Johns Hopkins University School of Medicine, 600 N. Wolfe Street, Osler 508, Baltimore, MD 21287, USA

Full list of author information is available at the end of the article Members of the HIV-COVID-19 Consortium are listed in

Acknowledgments. Many contributors helped with this work by enrolling their patients, and collecting data. Authors of this current manuscript are not listed in the following list.
}

disease severity at admission, and a number of underlying conditions (adjusted Hazard Ratio (aHR) 1.30, 95\% CI 1.24-1.36) [2]. Severe COVID-19 in the general population is associated with a hyperinflammatory syndrome similar to cytokine release syndrome (CRS) [3-5], thus, there have been several clinical trials of tocilizumab, an IL-6 receptor inhibitor (IL-6ri) approved for chimeric antigen receptor (CAR) T-cell-associated CRS [6-9]. One large randomized trial found that tocilizumab combined with corticosteroids led to a reduction in 28 day mortality in hypoxic patients with evidence of systemic inflammation $(\mathrm{CRP} \geq 75 \mathrm{mg} / \mathrm{L})$ [10]. Another trial found a reduction in organ support among ICU patients [9]. However, several smaller studies, particularly those enrolling before corticosteroids were in common use, have shown 
mixed results, and it remains unclear which patient population benefits most [11-14]. A meta-analysis of clinical trials of patients hospitalized for COVID-19 showed that treatment with an IL-6ri was associated with significantly lower 28-day all-cause mortality as compared to placebo or usual care (summary odds ratio (OR) 0.86 [95\% CI 0.79-0.95]; $\mathrm{p}=0.003$ ) [15], and tocilizumab is now part of the National Institutes of Health (NIH) and Infectious Diseases Society of America COVID-19 treatment guidance for patients hospitalized with progressive or severe COVID-19 and elevated inflammatory markers [16, 17]. But, as use of an IL-6ri can be associated with increased risk of secondary infections [18-21], guidelines recommend against their use in severely immunocompromised patients [6].

It is unclear whether PWH have increased risk for secondary infections or other adverse events after the use of IL-6ri for severe COVID-19. There are few reports of PWH receiving IL-6ri for COVID-19 [22, 23]. Here, we present 4 narrative case reports accompanied by a description of the outcomes of 18 patients with a diagnosis of HIV and PCR-confirmed COVID-19 treated with IL-6ri between April and June 2020. As data was retrieved from a pre-existing data base (The COVID-19 in PWH Registry [24]) with a different clinical question, the specific IL-6ri could not always be known. Additionally, cases in this series occurred prior to the publication of the RECOVERY trial (June 16, 2020), which showed that dexamethasone lowered 28-day mortality among hospitalized patients with COVID-19 [8], and prior to FDA approval of remdesivir (October, 22 2020) [25]. Therefore, corticosteroids and remdesivir were not routinely used, and effects arising from the combination of these drugs and IL-6ri would not be accounted for.

\section{Methods}

Data was combined from The COVID-19 in PWH Registry [24] and from internal chart review at the Johns Hopkins Hospital (JHU IRB00249576) between April and June 2020. The COVID-19 in PWH Registry is a multinational registry (Additional file 1: Supplemental Methods) which is sponsored by the University of Missouri, Columbia (The study was reviewed by the University of Missouri Institutional Review Board and considered to be exempt MU IRB 00000731, 00009014).

\section{Results}

Baseline characteristics from $18 \mathrm{PWH}$, including the 4 presented above, were collected across 8 centers in 3 countries between April 1, 2020 and June 30, 2020 and are presented in Table 1 . Median age was 60.5 years (29-76), and thirteen (72\%) patients were Black or Hispanic. At least 1 comorbid condition was present in $14(78 \%)$ patients, with HTN, DM, and CKD most

Table 1 Baseline characteristics of HIV patients receiving IL-6 inhibition for COVID-19 pneumonia

\begin{tabular}{|c|c|c|c|c|c|c|c|c|}
\hline Pt & Sex & Age & Race/ethnicity & Location & Most recent VL & CD4 & ART & Comorbid conditions \\
\hline 1 & $F$ & 60 & White, non-Hispanic & $\ln t$ & Undetectable & 600 & 2 NRTIs + PI + INSTI & COPD, CKD, Cirrhosis \\
\hline 2 & M & 39 & Hispanic, white & Int & Undetectable & 354 & 2 NRTIS + INSTI & HTN \\
\hline 3 & M & 74 & Black, non-Hispanic & MA & Undetectable & 238 & $2 \mathrm{NRTIS}+\mathrm{INSTI}$ & HTN, CKD, malignancy \\
\hline 4 & $F$ & 76 & Black, non-Hispanic & PA & Undetectable & 205 & 2 NRTIS + INSTI & HTN, DM, Asthma, CKD \\
\hline 5 & M & 61 & White, non-Hispanic & NY & 29 & 62 & $2 \mathrm{NRTIS}+\mathrm{INSTI}+\mathrm{CYP} 3 \mathrm{Ai}+\mathrm{PI}$ & Asthma \\
\hline 6 & M & 60 & Hispanic; non-white & NY & Undetectable & 1200 & $2 \mathrm{NRTIS}+\mathrm{INSTI}$ & None \\
\hline 7 & M & 59 & Hispanic; non-white & NY & Undetectable & 298 & NNRTI + INSTI & HTN, DM \\
\hline 8 & $\mathrm{~F}$ & 68 & Hispanic, non-white & NY & Undetectable & 186 & 2 NRTIS + INSTI & Cirrhosis \\
\hline 9 & M & 80 & Black. non-Hispanic & NY & Undetectable & 296 & 2 NRTIS + NNRTI & HTN, CKD \\
\hline 10 & $\mathrm{~F}$ & 39 & White, non-Hispanic & NY & Unknown & 85 & 2 NRTIS + INSTI & None \\
\hline 11 & M & 29 & Hispanic, non-white & NY & Undetectable & 465 & 2 NRTIS + INSTI & HTN \\
\hline 12 & M & 36 & Hispanic, white & $\mathrm{TX}$ & 24 & 328 & 2 NRTIS + NNRTI & None \\
\hline 13 & $M$ & 70 & White, non-Hispanic & $\mathrm{TX}$ & Undetectable & 260 & 2 NRTIS + INSTI & COPD, CKD \\
\hline 14 & $\mathrm{~F}$ & 57 & Black, non-Hispanic & PA & 84 & 250 & 2 NRTIS + INSTI & HTN, DM, CKD \\
\hline 15 & $M$ & 81 & Hispanic, non-white & $\mathrm{TX}$ & 91 & 345 & $2 \mathrm{NRTIS}+\mathrm{PI}$ & DM, malignancy \\
\hline 16 & M & 70 & White, non-Hispanic & $\mathrm{TX}$ & Undetectable & 260 & 2 NRTIS + NNRTI & COPD \\
\hline 17 & $M$ & 61 & Hispanic, non-white & NY & Undetectable & NR & 2 NRTIS + INSTI & HTN, CAD \\
\hline 18 & M & 57 & Black, non-Hispanic & MD & 326 & 111 & N/A & None \\
\hline
\end{tabular}

Pt patient, Int international, MA Massachusetts, PA Pennsylvania, NY New York, TX Texas, MD Maryland, VL viral load (1000 copies/mL), CD4 most recent documented absolute CD4 count (cells/mm [3]), COPD chronic obstructive pulmonary disease, CKD chronic kidney disease, HTN hypertension, DM diabetes mellitus, ART antiretroviral therapy, NRTI nucleoside reverse transcriptase inhibitor, NNRT non-nucleoside reverse transcriptase inhibitor, PI protease inhibitor, INSTI integrase strand transfer inhibitor, CYP3Ai CYP3A inhibitor, N/a new diagnosis not on treatment 
commonly reported. HIV VL was $<50$ copies/mL in 14 (82\%), unknown in 1 , and $>200$ copies $/ \mathrm{mL}$ in 1 patient. CD4 counts were $<200$ cells $/ \mathrm{mm}^{3}$ in $4(22 \%)$ patients, unknown for 1 , and $>200$ cells $/ \mathrm{mm}^{3}$ in 13 . All patients with previously diagnosed HIV were on antiretroviral therapy throughout their admissions; the patient with HIV/AIDS newly diagnosed during his ICU stay for COVID was never started on ART.

SARS-CoV2 was confirmed by PCR in all patients. The administered IL-6ri was tocilizumab in 4, sarilumab in 6, and an unknown IL-6ri in 8 patients (Table 2). Timing of administration was reported for 4 patients, all of them treated within 10 days of being hospitalized (3-10 days of hospitalization), and unknown for the remaining patients. Only 2 patients received corticosteroids, while none received remdesivir. On admission, median CRP was $104(7-207) \mathrm{mg} / \mathrm{L}$, with 9 (50\%) patients $>100 \mathrm{mg} / \mathrm{L}$. Median D-dimer was $0.26(0-4) \mathrm{mg} /$ $\mathrm{mL}-5$ patients presented with $\mathrm{D}$-dimer of $\leq 0.002 \mathrm{mg} /$ $\mathrm{mL}, 12>0.18 \mathrm{mg} / \mathrm{L}$, and 1 without a reported value. Presenting absolute lymphocyte count was $<1.0 \times 10^{3}$ cells/ $\mu \mathrm{L}$ in 7 (39\%) patients. Secondary or opportunistic infections were treated in $8(44 \%)$ patients, all admitted to the ICU, and 4 died. A specific organism was identified in 5 patients, including Stenotrophomonas, S. aureus, C. albicans, E. cloacae, $K$. aerogenes respiratory infections, and disseminated $V Z V$ (case 1). It was unclear whether these infections arose before or after IL-6ri in at least 3 of these cases. Of the 4 patients with CD 4 counts $<200$ cells/ $\mathrm{mm}^{3}$, three were treated for secondary infection, with 2 confirmed organisms and 2 deaths, while in 13 patients with CD4 counts $>200$ cells $/ \mathrm{mm}^{3}, 5$ were treated for secondary infection, with 3 confirmed organisms and 5 deaths. Overall, ICU admission was required for 12 (67\%) patients, including 3 of 4 subjects with CD4 counts $<200$ cells, and 3 of 6 patients older than 65 . Eleven (61\%) patients required mechanical ventilation, and death occurred in 7 (39\%) patients.

\section{Case presentations \\ Case 1}

A 57-year-old man with no known medical conditions presented to the emergency department (ED) with 1 week of fevers, dyspnea, and fatigue. He was hypoxic, requiring supplemental oxygen by nasal cannula (NC). SARS-COV-2 by PCR returned positive, ceftriaxone and azithromycin were initiated, and he was hospitalized. On admission IL-6 was $92 \mathrm{pg} / \mathrm{mL}$ (ref. $<10 \mathrm{pg} / \mathrm{mL}$ ), CRP $7 \mathrm{mg} / \mathrm{L}$ (ref.: $<0.5 \mathrm{mg} / \mathrm{L}$ ), and D-dimer $1 \mathrm{mg} / \mathrm{L}$ (ref.: $0.19-0.79 \mathrm{mg} / \mathrm{L}$ ). On day 4 of hospitalization, hypoxemia progressed requiring intubation, and he received tocilizumab $480 \mathrm{mg}$, but not corticosteroids or remdesivir. Over the next 3 days he developed refractory hypoxemia and shock managed with prone positioning and vasopressors. Multisystem organ failure progressed, and he required continuous veno-venous hemodialysis. On day 8 of hospitalization, he developed transaminitis with AST 337 IU/L, ALT 242, alkaline phosphatase 181 that persisted. Additional infectious workup on Day 40 of hospitalization revealed a new diagnosis of AIDS (positive HIV-1 antibody, CD4 count 111 cells/ $\mathrm{mm}^{3}$; HIV-RNA 326,000 copies $/ \mathrm{mL}$ ) and presumed late latent syphilis (positive anti-treponemal antibody, negative RPR, positive TPPA). He was treated with 3 once-weekly doses of Benzathine penicillin. After 47 days of hospitalization he was noted to have a diffuse papular rash which became vesicular, and was unroofed. PCR testing of these lesions was positive for Varicella Zoster Virus (VZV), confirming a diagnosis of disseminated VZV. Concurrently, the patient's hypotension became refractory to vasopressors and maximal supportive measures, and care was ultimately withdrawn on hospital day 49. Autopsy was declined.

\section{Case 2}

A 74-year-old man with known HIV (CD4 234 cells/ $\mathrm{mm}^{3}$, VL undetectable, on Bictegravir/Emtricitabine/ Tenofovir alafenamide), dementia, hypertension (HTN), stage 3 chronic kidney disease (CKD), and presumed gastric carcinoma treated with one dose of pembrolizumab, presented to the ED with abdominal pain, fatigue, and altered mental status. Presenting oxygen saturation was $97 \%$ on room air (RA) but worsened, requiring supplemental oxygen. He tested positive for SARS-CoV-2 by PCR. Initial computed tomography (CT) of the chest revealed bilateral opacities, pleural effusions, and presumed worsening metastatic spread of malignancy. On admission, IL-6 was $68 \mathrm{pg} / \mathrm{mL}$, CRP $94 \mathrm{mg} / \mathrm{L}$, and D-dimer $4 \mathrm{mg} / \mathrm{mL}$. He was not eligible for remdesivir due to renal function; corticosteroids were not given. On day 9 of hospitalization, with persistent hypoxia and intermittent fevers, he received $400 \mathrm{mg}$ tocilizumab. On day 16 , hypoxia progressed, requiring oxygen via high flow nasal cannula (HFNC). A repeat CT scan revealed worsening pleural effusions. Given his cancer diagnosis and poor prognosis, he was transitioned to comfort measures the following day and died of multisystem organ failure on day 21 of his hospitalization.

\section{Case 3}

A 60-year-old woman with prior intravenous drug use, HIV (CD4 600 cells $/ \mathrm{mm}^{3}$, undetectable HIV VL, on Abacavir/Lamivudine + Raltegravir), recently cured hepatitis $C$ virus, cirrhosis, and Stage 1 CKD presented to the ED with cough, headache, dyspnea, fevers, sore throat, myalgias, and fatigue. She was hypoxic, initially 


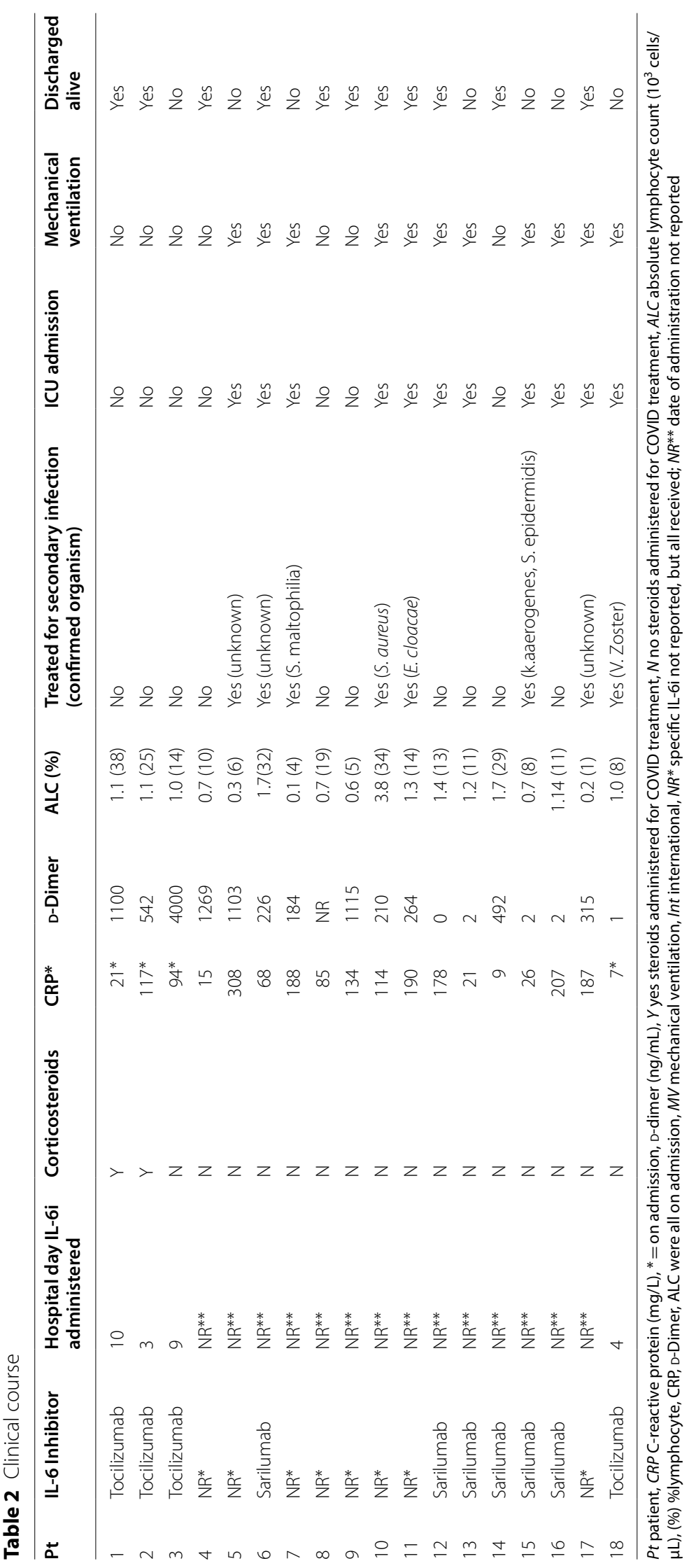


requiring 3-5 L/min of oxygen via $\mathrm{NC}$, but soon escalated to Bilevel Positive Airway Pressure (BiPAP). Initial chest X-ray (CXR) revealed multifocal opacities, and contrasted chest $\mathrm{CT}$ was suggestive of viral pneumonia without a pulmonary embolism. SARS-CoV-2 RT-PCR was positive, she was initiated on ceftriaxone and azithromycin and hospitalized. On admission CRP was $21 \mathrm{mg} / \mathrm{L}$, D-dimer $1.1 \mathrm{mg} / \mathrm{mL}$, and absolute lymphocyte count $0.9 \times 10^{3}$ cells $/ \mu \mathrm{L}$. She was treated with corticosteroids, but not remdesivir. On Hospital Day 2, she received $400 \mathrm{mg}$ of tocilizumab and initiated 4 days of methylprednisolone and hydroxychloroquine. On day 8 of hospitalization, D-dimer peaked at $2023 \mathrm{ng} / \mathrm{mL}$. Thereafter, she improved and was discharged to a continuing care facility on day 27 of her hospitalization with complete clinical recovery.

\section{Case 4}

A 39-year-old man with HIV (CD4 354 cells $/ \mathrm{mm}^{3}$, HIV VL undetectable, on Elvitegravir/Cobicistat/Emtricitabine/Tenofovir-alafenamide plus Darunavir $800 \mathrm{mg}$ daily) and HTN presented with cough, dyspnea, fevers, sore throat, myalgias, and fatigue. In the ED, he was tachypneic, but saturating $96 \%$ on room air. SARS-CoV-2 was positive by RT-PCR. He was initiated on ceftriaxone, azithromycin, and hydroxychloroquine, and hospitalized. CXR revealed multifocal opacities, and on admission IL-6 was $73 \mathrm{pg} / \mathrm{mL}$, CRP $117 \mathrm{mg} / \mathrm{L}$, D-dimer $0.5 \mathrm{mg} / \mathrm{mL}$, fibrinogen $984 \mathrm{mg} / \mathrm{dL}$, and absolute lymphocyte count $0.8 \times 10^{3}$ cells $/ \mu \mathrm{L}$. Over the next 2 days, hypoxia worsened and he required HFNC $\mathrm{O}_{2}$. IL-6 and D-dimer rose to $296 \mathrm{pg} / \mathrm{mL}$ and $843 \mathrm{ng} / \mathrm{mL}$, respectively. On day 3 of hospitalization, he received $400 \mathrm{mg}$ tocilizumab and initiated 4 days of methylprednisolone. He improved clinically and was discharged home on RA on hospital day 9.

\section{Discussion}

Here we report one of the first case series of PWH who received IL-6ri for severe COVID-19, comprising 18 individuals. Most patients did not receive corticosteroids or remdesivir because of local standard of care in this time period early in the pandemic. $77 \%$ of the patients in this series had at least one co-morbid condition, increasing their risk for worse outcomes. The first diagnosis of HIV in a patient with AIDS at the time of ICU admission for COVID-19 (also previously reported) [22, 23] makes a compelling case for routine HIV screening on all patients hospitalized with severe or critical COVID-19.

All previously diagnosed patients were on anti-retroviral therapy, and HIV VL was $<50$ copies $/ \mathrm{mL}$ in $82 \%$ of patients, with CD4 counts over 200 cells $/ \mathrm{mm}^{3}$ in $78 \%$ of patients. Still, there was a high prevalence of secondary infections. Although we are uncertain when the infections were present compared to administration of IL-6ri, clinical outcomes in the overall cohort and particularly among patients treated for secondary infection were poor, with death occurring in half of patients treated for infection. This is unsurprising given the baseline prognostic indicators, timing of this study during early pandemic surge [26], and the lack of contemporaneously available efficacious treatments such as corticosteroids and remdesivir [6].

As one of the only series of PWH receiving IL-6ri, this study adds a critical clinical perspective for providers treating PWH and COVID-19. The series draws from an ethnically diverse population from 8 hospitals with a high prevalence of co-morbid conditions. Confirmation of secondary infecting organisms was available for $64 \%$ of the patients treated for secondary infections, adding to an understanding of the diversity of secondary infections (along with COVID-19) which might behave opportunistically after treatment with IL-6ri.

As a case series, this series is retrospective, and the number of patients is few. Cases accrued prior to corticosteroids and remdesivir becoming standard of care for the treatment of COVID-19; only 2 patients received corticosteroids while none received remdesivir. Additionally, institutional policies on criteria and timing of administration of IL-6ri varied widely by site, and were not always clearly documented. Finally, data were obtained from a database that did not include specification of which exact IL-6ri was used, and this information was therefore not always obtainable. As head-to-head comparisons of one IL-6ri verses another are scarce, the impact of this limitation on the results is unclear.

\section{Conclusion}

There is currently a lack of data on how best to individualize care for PWH with COVID-19. Immune-based therapy for COVID-19, while potentially lifesaving, may have potential for harm in certain immunocompromised patients, such as PWH with $\mathrm{CD} 4<200$ cells $/ \mathrm{mm}^{3}$ or AIDS. Given the tens of millions of PWH living in settings with poor access to COVID-19 vaccines, this series highlights the need for further high-quality investigations of IL-6ri in PWH who are hospitalized with COVID-19.

\section{Supplementary Information}

The online version contains supplementary material available at https://doi. org/10.1186/s12981-022-00430-x.

Additional file 1: Supplementary methods

\section{Acknowledgements}

New York University Grossman School of Medicine, NY: Savannah KarmenTuohy, BS; Philip M. Carlucci, BS; loannis M. Zacharioudakis, MD; Joseph Rahimian, MD; Fainareti N Zervou, MD; Gabriel Rebick, MD; Anna Stachel, Ph.D.; Shini 
Tang, BS; Dan Ding, MA. Johns Hopkins University, MD: Joyce L Jones, MD, MS; Jason E. Farley, Ph.D., MPH; Kelly E. Dooley, MD, Ph.D. Barbara E. Wilgus, MSN, CRNP; Michael Sanchez, DNP, CRNP, FNP-BC, FAANP. UT Southwestern Medical Center/Parkland Memorial Hospital, TX, USA: Jeremy Chow, MD, MS; Ellen Kitchell, MD; Shannon Koh, MD; Daniel Maxwell, MD; Abby Lau, MD; Shamika Brooks APRN, FNP-C, AAHIVS; Jessica Chu, PA-C; Joshua Estrada, PA-C, AAHIVS, and Susana M. Lazarte, MD. Wellstar Kennestone Regional Medical Center, GA: Folasade Arinze, MD, MPH; Adero Francis, MD; Neha Paranjape, MD, MPH. Brigham and Women's Hospital, MA: Paul E. Sax, MD. Vanderbilt University Medical Center, TN: Celestine N. Wanjalla MD, PhD; Asghar N. Kheshti, Samuel Bailin MD, John R. Koethe MD, Sean G. Kelly MD, Stephen P. Raffanti MD. Baylor College of Medicine, TX: Shital M. Patel, MD, MS; Teena Huan Xu, MD; Melanie Goebel, MD. Infectious Diseases and Fight AIDS Foundation, University Hospital Germans Trias, Badalona, Spain. Puerta de Hierro Majadahonda University Hospital, Madrid, Spain: Alberto Díaz-De Santiago, MD, Ph.D. Santa Clara Valley Medical Center, CA: Manoj Ray, MD; Carol Hu, MD. Saint Michael's Medical Center, NJ: Jihad Slim, MD. Tower Health, PA: Ann Marie Porreca Kratz, PharmD, BCPS, BCIDP. Temple University Health System, PA: David E. Koren, PharmD; Kayla Hiryak, PharmD. The University of Arkansas for Medical Sciences, AR: Brannon Hill, PharmD; Ryan K Dare, MD, MS; Stacie Bordelon, PharmD, AAHIVP; Brett Bailey, PharmD. University of Maryland, MD: John W. Baddley, MD, MSPH. University of Kansas Medical Center, KS: D. Matthew Shoemaker, DO. AMITA Health Saint Francis Hospital, L: Guillermo Rodriguez-Nava, MD. Mayo Clinic College of Medicine, Rochester, MN: FNU Shweta, MBBS. The University of California, San Francisco Medical Center, CA: Carolyn Chu, MD, MSc; Catherine Pearson, MD. The Polyclinic, WA: Amy Treakle, MD. Case Western Reserve University, OH: Jennifer J. Furin, MD, Ph.D. MetroHealth Medical Center, $\mathrm{OH}$ : Milana Bogorodskaya, MD. Hackensack University Medical Center, NJ: Samit Desai, MD. Hurley Medical Center/Michigan State University College of Human Medicine, MI: Danielle Osterholzer, MD. San Joaquin General Hospital, CA: Jered Arquiette, PharmD, BCPS-AQ ID. University of Washington, WA: Emily S. Ford, MD. University of Maryland Medical Center Midtown Campus, MD: Patrick R. Ching, MD, MPH. Alexandra Hospital, National University Health System (NUHS), Singapore: Louisa Sun, MBBS, MRCP. Washington University, MO. Advocate Aurora St. Luke's Medical Center, WI: Brian P. Buggy, MD. Indiana University Health, IN: Amir Tirmizi, MD. Mount Sinai Hospital, IL: Sarah Argentine, NP. SOVAH health, VA: Balaji Desai, MD. Icahn School of Medicine at Mount Sinai, NY: Talia H. Swartz, MD, PhD. Callen-Lorde Community Health: Dusty Latimer, PA-C. University of Missouri, Columbia: Maraya Camazine, MS; Andres Bran, MD.

The authors would like to acknowledge the HIV-COVID-19 consortium and the many contributors who helped with this work by enrolling their patients, and collecting data.

\section{Authors' contributions}

As principal investigator, DD had full access to all the data in the study and takes responsibility for the integrity of the data and the accuracy of the data analysis. Study concept and design: SJM, EDW. Acquisition of data: All authors. Analysis and interpretation of data: All authors. Drafting of the manuscript: SJM, AAR Antar, DD, EDWeld. All authors read and approved the final manuscript.

\section{Funding}

This research received no specific grant from any funding agency in the public, commercial, or not-for-profit sectors.

\section{Availability of data and materials}

Raw data were generated from The COVID-19 in PWH Registry [24] and from internal chart review at the Johns Hopkins Hospital (JHU IRB00249576). Derived data supporting the findings of this study are available from the corresponding author EDW on request.

\section{Declarations}

\section{Ethics approval and consent to participate}

Data was combined from The COVID-19 in PWH Registry [24] and from internal chart review at the Johns Hopkins Hospital (JHU IRB00249576) between April and June 2020. The COVID-19 in PWH Registry is a multi-national registry (Additional file 1: Supplemental Methods) which is sponsored by the University of Missouri, Columbia (The study was reviewed by the University of Missouri Institutional Review Board and considered to be exempt MU IRB $00000731,00009014)$. Anonymized patient data were collected without the need for informed consent.

\section{Consent for publication}

The authors agree to deliver to the responsible editor(s) on a date to be agreed upon the manuscript created according to the Instructions for Authors.

\section{Competing interests}

The authors have no competing interests to disclose.

\section{Author details}

${ }^{1}$ Department of Medicine, The Johns Hopkins University School of Medicine, 600 N. Wolfe Street, Osler 508, Baltimore, MD 21287, USA. ${ }^{2}$ Critical Care Medicine Department, National Institutes of Health, Bethesa, USA. ${ }^{3}$ University of Missouri-Columbia School of Medicine, Columbia, USA. ${ }^{4}$ Infectious Diseases and Fight AIDS Foundation, University Hospital Germans Trias, Badalona, Spain. ${ }^{5}$ Department of Infectious Diseases, Brigham and Women's Hospital, Boston, USA. ${ }^{6}$ Department of Medicine, University of Missouri, Columbia, USA.

Received: 16 November 2021 Accepted: 22 January 2022

Published online: 11 February 2022

\section{References}

1. Eisinger RW, Lerner AM, Fauci AS. HIV/AIDS in the era of Coronavirus 2019: a juxtaposition of 2 pandemics. J Infect Dis. 2021;224:1455-61.

2. WHO. Clinical features and prognostic factors of COVID-19 in people living with HIV hospitalized with suspected or confirmed SARS-CoV-2 infection. 2021

3. Chen X, Zhao B, Qu Y, et al. Detectable serum severe acute respiratory syndrome coronavirus 2 viral load (RNAemia) is closely correlated with drastically elevated interleukin 6 level in critically ill patients with coronavirus disease 2019. Clin Infect Dis. 2020;71(8):1937-42.

4. Del Valle DM, Kim-Schulze S, Huang HH, et al. An inflammatory cytokine signature predicts COVID-19 severity and survival. Nat Med. 2020;26(10):1636-43.

5. Herold T, Jurinovic V, Arnreich C, et al. Elevated levels of IL-6 and CRP predict the need for mechanical ventilation in COVID-19. J Allergy Clin Immunol. 2020;146(1):128-136e124.

6. COVID-19 Treatment Guidelines Panel. Coronavirus Disease 2019 (COVID19) Treatment Guidelines. National Institutes of Health. 2021.

7. Bhimraj A, Morgan RL, Shumaker AH, et al. Infectious Diseases Society of America Guidelines on the Treatment and Management of Patients with COVID-19. Clin Infect Dis. 2020. [p 22, referenced 6.20.2021]

8. Group RC, Horby P, Lim WS, et al. Dexamethasone in hospitalized patients with COVID-19. N Engl J Med. 2021;384(8):693-704.

9. Investigators R-C, Gordon AC, Mouncey PR, et al. Interleukin-6 receptor antagonists in critically ill patients with COVID-19. N Engl J Med. 2021;385:1147.

10. Group RC. Tocilizumab in patients admitted to hospital with COVID-19 (RECOVERY): a randomised, controlled, open-label, platform trial. Lancet. 2021;397(10285):1637-45.

11. Rosas 10 , Brau N, Waters $M$, et al. Tocilizumab in hospitalized patients with severe COVID-19 pneumonia. N Engl J Med. 2021;383:1504-16.

12. Salama C, Han J, Yau L, et al. Tocilizumab in patients hospitalized with COVID-19 pneumonia. N Engl J Med. 2021;384(1):20-30.

13. Salvarani C, Dolci G, Massari M, et al. Effect of tocilizumab vs standard care on clinical worsening in patients hospitalized with COVID-19 pneumonia: a randomized clinical trial. JAMA Intern Med. 2021;181(1):24-31.

14. Stone JH, Frigault MJ, Serling-Boyd NJ, et al. Efficacy of tocilizumab in patients hospitalized with COVID-19. N Engl J Med. 2020;383(24):2333-44.

15. Group RW, Shankar-Hari M, Vale CL, et al. Association between administration of IL-6 antagonists and mortality among patients hospitalized for COVID-19: a meta-analysis. JAMA. 2021;326:499-518.

16. Guidelines NC-T. Interleukin-6 Inhibitors. https://www.covid19treatmen tguidelines.nih.gov/therapies/immunomodulators/interleukin-6-inhib itors/2021. Accessed 20 June 2021. 
17. IDSA Guidelines on the Treatment and Management of Patients with COVID-19. In: America IDSo, ed. https://www.idsociety.org/practice-guide line/covid-19-guideline-treatment-and-management/2021.

18. Emery P, Keystone E, Tony HP, et al. IL-6 receptor inhibition with tocilizumab improves treatment outcomes in patients with rheumatoid arthritis refractory to anti-tumour necrosis factor biologicals: results from a 24-week multicentre randomised placebo-controlled trial. Ann Rheum Dis. 2008;67(11):1516-23.

19. Schiff $M H$, Kremer JM, Jahreis $A$, Vernon $E$, Isaacs JD, van Vollenhoven RF. Integrated safety in tocilizumab clinical trials. Arthritis Res Ther. 2011;13(5):R141.

20. Guaraldi G, Meschiari M, Cozzi-Lepri A, et al. Tocilizumab in patients with severe COVID-19: a retrospective cohort study. Lancet Rheumatol. 2020;2(8):e474-84.

21. Somers EC, Eschenauer GA, Troost JP, et al. Tocilizumab for treatment of mechanically ventilated patients with COVID-19. Clin Infect Dis. 2021;73(2):e445-54.

22. Liu A, Wei J, XuY, et al. Immune characteristics of human immunodeficiency virus/severe acute respiratory syndrome coronavirus 2 coinfection: a case report and mini-review. SAGE Open Med Case Rep. 2021;9:2050313X211014958.

23. Zhang JC, Yu XH, Ding XH, et al. New HIV diagnoses in patients with COVID-19: two case reports and a brief literature review. BMC Infect Dis. 2020;20(1):771.

24. Dandachi D, Geiger G, Montgomery MW, et al. Characteristics, comorbidities, and outcomes in a multicenter registry of patients with HIV and coronavirus disease-19. Clin Infect Dis. 2020;73(7):e1964-72.

25. VEKLURY (remdesivir) for injection, for intravenous use VEKLURY (remdesivir) injection, for intravenous use Initial U.S. Approval: 2020. https://www. accessdata.fda.gov/drugsatfda_docs/label/2020/2147870rig1 s000lbl.pdf. Published 2020. Accessed 20 Sept 2021.

26. Kadri SS, Sun J, Lawandi A, et al. Association between caseload surge and COVID-19 survival in 558 U.S. hospitals, March to August 2020. Ann Intern Med. 2021;174:1240-51.

\section{Publisher's Note}

Springer Nature remains neutral with regard to jurisdictional claims in published maps and institutional affiliations.

Ready to submit your research? Choose BMC and benefit from:

- fast, convenient online submission

- thorough peer review by experienced researchers in your field

- rapid publication on acceptance

- support for research data, including large and complex data types

- gold Open Access which fosters wider collaboration and increased citations

- maximum visibility for your research: over $100 \mathrm{M}$ website views per year

At BMC, research is always in progress.

Learn more biomedcentral.com/submissions 